\title{
Laypeople Can Predict Which Social Science Studies Replicate
}

\author{
Suzanne Hoogeveen*, Alexandra Sarafoglou*, Eric-Jan Wagenmakers \\ Department of Psychology, University of Amsterdam, The Netherlands \\ *The first two authors contributed equally to this work
}

\begin{abstract}
Large-scale collaborative projects recently demonstrated that several key findings from the social-science literature could not be replicated successfully. Here, we assess the extent to which a finding's replication success relates to its intuitive plausibility. Each of 27 high-profile social science findings was evaluated by 233 people without a $\mathrm{PhD}$ in psychology. Results showed that these laypeople predicted replication success with above-chance accuracy (i.e., 59\%). In addition, when participants were informed about the strength of evidence from the original studies, this boosted their prediction performance to $67 \%$. We discuss the prediction patterns and apply signal detection theory to disentangle detection ability from response bias. Our study suggests that laypeople's predictions contain useful information for assessing the probability that a given finding will replicate successfully.

Keywords: Open Science, Meta-Science, Replication Crisis, Prediction Survey
\end{abstract}

Recent work has suggested that the replicability of social-science research may be disturbingly low (Baker, 2016). For instance, several systematic high-powered replication projects have demonstrated successful replication rates ranging from 36\% (Open Science Collaboration, 2015), 50\% (Klein et al., 2018), to 62\% (Camerer et al., 2018), to $85 \%$ (Klein, Ratliff, Vianello, Adams, et al., 2014). These low replication rates have been explained by several factors that operate at different levels. At the level of the scientific field as a whole, problems include publication bias (Francis, 2013) and perverse incentive structures (Giner-Sorolla, 2012). At the level of individual studies, problems concern low

Correspondence concerning this article should be addressed to: Suzanne Hoogeveen, Nieuwe Achtergracht 129B, 1001 NK Amsterdam, The Netherlands, E-mail: suzanne.j.hoogeveen@gmail.com.

Preregistration, data and analysis code are provided at https://osf.io/x72cy/. 
statistical power (Button et al., 2013; Ioannidis, 2005) and questionable research practices such as data-driven flexibility in statistical analysis (i.e., significance seeking; John, Loewenstein, \& Prelec, 2012; Simmons, Nelson, \& Simonsohn, 2011; Wagenmakers, Wetzels, Borsboom, \& van der Maas, 2011). Here we focus on yet another problem that has recently been associated with poor replicability: the a priori implausibility of the research hypothesis (Benjamin et al., 2018; Ioannidis, 2005).

If the a priori implausibility of the research hypothesis is indicative of replication success, then replication outcomes can be reliably predicted from a brief description of the hypothesis at hand. Indeed, results from recent surveys and prediction markets demonstrated that researchers (i.e., experts) in psychology and related social sciences can anticipate replication outcomes with above-chance accuracy - as a group, experts correctly predicted the replication outcomes for $58 \%, 67 \%$, and $86 \%$ of the studies included in the Reproducibility Project: Psychology, the Many Labs 2 project (ML2), and the Social Science Replication project (SSRP) respectively (Camerer et al., 2018; Dreber et al., 2015; Forsell et al., 2018). These surveys and prediction markets involved forecasters with a $\mathrm{PhD}$ in the social sciences (e.g., psychology, economics). In addition, the forecasters in Forsell et al. (2018) and Camerer et al. (2018) had been provided with statistical information from the original studies, including $p$-values, effect sizes, and/or sample sizes. These findings raise two key questions about anticipated replicability: First, do forecasters need to be social science experts to predict replication outcomes with above-chance accuracy? Second, are forecasters' predictions driven by intuitions about empirical plausibility alone or also influenced by statistical information about the original effect?

In this study, our primary aim was to investigate whether and to what extent accurate predictions of replicability can be generated by people without a $\mathrm{PhD}$ in psychology or other professional background in the social sciences (i.e., laypeople) and without access to the statistical evidence obtained in the original study. Laypeople may be able to reliably evaluate the plausibility (and hence replicability) of research hypotheses, even without access to relevant statistical information or in-depth knowledge of the literature - after all, social science concerns itself with constructs that are often accessible and interesting to a lay audience (Milkman \& Berger, 2014). Consequently, when presented with a non-technical description of a study's topic, operationalization and result, laypeople may well be able to produce accurate replicability forecasts. For example, consider a nontechnical description of Kidd and Castano's (2013) research hypothesis:

"Can reading literary fiction improve people's understanding of other people's emotions? Participants read a short text passage. In one group, the text passage was literary fiction. In the other group, the text passage was non-fiction. Afterwards, participants had to identify people's expressed emotion (e.g., happy, 
angry) based on images of the eyes only. Participants were better at correctly recognizing the emotion after reading literary fiction."

A general understanding of the concepts (e.g., literary fiction, emotions) and proposed relation between those concepts (e.g., reading literary fiction improves emotion recognition) may suffice to form intuitions about plausibility that match the (eventual) empirical evidence. The accuracy of such intuitions can be gauged by comparing laypeople's predictions with the empirical outcome - hence, for this study, we selected 27 high-profile findings that have recently been submitted to high-powered replication attempts (Camerer et al., 2018; Klein et al., 2018).

If laypeople can indeed make accurate predictions about replicability, these predictions may supplement theoretical considerations concerning the selection of candidate studies for replication projects. Given limited resources, laypeople's predictions concerning replicability could be used to define the subset of studies for which one can expect to learn the most from the data. In other words, researchers could use laypeople's predictions as input to assess information gain in a quantitative decision-making framework for replication (Hardwicke, Tessler, Peloquin, \& Frank, 2018; MacKay, 1992). This framework follows the intuition that -for original studies with surprising effects (i.e., low plausibility) or small sample sizes (i.e., little evidence) - replications can bring about considerable informational gain (Klein, Ratliff, Vianello, Adams Jr, et al., 2014).

More generally, if even laypeople can to a large extent correctly pick out the unreplicable findings, this suggests that researchers should be cautious when making bold claims on the basis of studies with risky and counterintuitive hypotheses. Laypeople's adequate predictions of replicability may thus provide empirical support for a culture change that emphasizes robustness and 'truth' over novelty and 'sexiness' (Dovidio, 2016; Nosek, Spies, \& Motyl, 2012; Giner-Sorolla, 2012). When extended to novel hypotheses, laypeople's skepticism may even serve as a 'red flag', prompting researchers to go the extra mile to convince their audience -laypeople and peers alike- of the plausibility of their research claim (e.g., by using larger samples, engaging in Registered Reports, or setting a higher bar for evidence; see Benjamin et al., 2018; Chambers, 2013).

The secondary aim of the current study was to assess the extent to which the inclusion of information about the strength of the evidence obtained in an original study improves laypeople's prediction performance. In contrast to the investigations of experts' prediction accuracy by Camerer et al. (2018) and Forsell et al. (2018), we used Bayes factors rather than $p$-values and effect sizes to quantify the evidence in the original studies (Jeffreys, 1961; Kass \& Raftery, 1995).

We preregistered the following hypotheses: First, we hypothesized that, on the basis of assessments of the a priori plausibility of the research hypotheses at hand, (1a) laypeople 
can predict the replicability of empirical studies with above-chance accuracy, and (1b) their confidence is associated with the magnitude of the effects of interest in the replication study. The former hypothesis would be supported by prediction accuracy above $50 \%$ and the latter by a positive correlation between people's confidence in replicability and the effect size in the replication. In addition, we hypothesized that (2) the inclusion of information on the strength of the original evidence (i.e., the Bayes factor) would improve prediction performance.

\section{Disclosures}

\section{Data, materials, and preregistration}

The current study was preregistered on the Open Science Framework by means of a timestamped PDF; readers can access the preregistration, as well as all materials, reanalyses of the original studies, the anonymized raw and processed data (including relevant documentation for the data of ML2 and SSRP), and the R code to conduct all confirmatory and exploratory analyses (including all figures), in our OSF folder at: https://osf .io/x72cy/. Any deviations from the preregistration are mentioned in this manuscript.

\section{Supplemental Material}

In the online Supplemental Material we provide additional details on the methods and additional exploratory analyses. Specifically, the online supplement presents details on the Bayesian reanalyses of the original studies, the sampling plan, and the statistical models and prior specifications; includes tables with the descriptions (in English and Dutch) of the original studies as presented to the participants; and reports two additional exploratory analyses. The first of these analyses concerns the accuracy of predictions derived from the Bayes factors alone, without human evaluation, and the second analysis is a Bayesian logistic regression model that includes random effects for both participants and studies.

\section{Reporting}

We report how we determined our sample size, all data exclusions, all manipulations, and all measures in the study.

\section{Ethical approval}

The study was approved by the local ethics board of the University of Amsterdam and all participants were treated in accordance with the Declaration of Helsinki. 


\section{Method}

\section{Participants}

In total, we obtained data from 257 participants, who were recruited from the online platform Amazon Mechanical Turk $(n=83)$, the online participant pool of first-year psychology students at the University of Amsterdam ( $n=138)$, and social-media platforms such as Facebook $(n=36)$. Participants from MTurk received a financial compensation, University of Amsterdam students received research credits, and participants from social media were given the opportunity to enter a raffle for a voucher from a Dutch web-shop. After exclusions (see below), the final sample consisted of 233 participants, with 123 participants in the Description Only condition and 110 participants in the Description Plus Evidence condition.

\section{Sampling Plan}

We determined that we would need a minimum number of 103 observations per condition to achieve an $80 \%$ probability of obtaining strong evidence (i.e., a Bayes factor $>10$ ) in favor of our experimental hypothesis, assuming a medium effect size of $\delta=0.5$, a default Cauchy prior with a scale parameter of $r=0.707$, and a study design comparing two independent groups (i.e., a $t$-test). As preregistered, data collection continued after the minimum number of participants was reached (i.e., 103 in each condition), until the preestablished data collection termination date of April 22, 2019.

\section{Materials}

Participants were presented with 27 studies, a subset of the studies included in the SSRP (Camerer et al., 2018) and the ML2 project (Klein et al., 2018).

Study Selection. In the Description Plus Evidence condition, participants were provided with study descriptions accompanied by Bayes factors indicating the strength of the original evidence. Therefore, one of the main criteria for inclusion in the set of studies was that the original analysis allowed for a Bayesian reanalysis using the Summary Stats module in JASP (JASP Team, 2019); that is, the main analysis had to have been conducted using a paired-samples or independent samples $t$-test, a correlation test, or a binomial test. ${ }^{1}$ Details about the reanalyses are provided in the Supplemental Material. We subsequently checked whether the percentage of successful vs. unsuccessful replications in our subset was similar to the percentage in ML2 and the SSRP (i.e., 50\% and 62\%). This was the case; our subset included 14 successful and 13 unsuccessful replications (i.e., $52 \%$ successful).

\footnotetext{
${ }^{1}$ For some studies, the original articles reported $F$-values derived from ANOVA designs, but as the crucial comparison was between only two groups, we converted the respective $F$-value to a $t$-value, which was then entered in the Summary Stats module in JASP.
} 
Presentation of Studies. The materials for each study included a short description of the research question, its operationalization, and the key finding. These descriptions were inspired by those provided in the SSRP and ML2, but were rephrased to be comprehensible by laypeople. In the Description Only condition, solely these descriptive texts were provided; in the Description Plus Evidence condition, the Bayes factor and its verbal interpretation (e.g., "moderate evidence") for the original study were added to the description of each study. The verbal interpretations were based on a classification scheme proposed by Jeffreys (1939) and adjusted by M. D. Lee and Wagenmakers (2013, p. 105). These verbal labels were added to help participants interpret the Bayes factors, because the concept of evidence ratios might be difficult or ambiguous for laypeople (Etz, Bartlema, Vanpaemel, Wagenmakers, \& Morey, 2019). To prevent participants from reading about the replication outcomes of the original studies during the survey itself, we ensured that the descriptions did not contain identifying information, such as the names of the authors, the study titles, or any direct quotes. In addition to the 27 study descriptions, the survey also included one bogus item as an attention check. In the description of this study participants were instructed to answer "no" to the question of whether the study would replicate and to indicate a confidence of $75 \%$ in this prediction. Participants from the Netherlands could choose to read the study descriptions in English or Dutch. The translation of the descriptions from English into Dutch was assisted by the online translation software DeepL (www.deepl.com/translator).

\section{Procedure}

The survey was generated using the online survey software Qualtrics (www .qualtrics.com). Participants were randomly assigned to the Description Only or the Description Plus Evidence condition. First, they read an explanation of the term 'replication' and its relevance in science: "You will be asked whether you think that the described study will replicate. This means: if an independent lab will do this study again with a large number of participants, using the same materials, will they find convincing evidence for the same effect? If the effect really exists, it should be found by a different lab. However, it seems that not all studies can be replicated, because some results are based on coincidence, or poorly designed or executed studies." Participants in the Description Plus Evidence condition additionally received a short explanation of the Bayes factor and the commonly used verbal cateogries for interpreting the strength of evidence they indicate (M. D. Lee \& Wagenmakers, 2013, p. 105). The explanation of the Bayes factor was as follows: "A Bayes factor (BF) is the degree to which evidence is found for the existence of the effect, based on the data at hand. For instance, if $\mathrm{BF}=2$, the data suggest that it is 2 times more likely that the effect is 
present, than that there is no effect." ${ }^{2}$

After the instructions, participants were presented with the 27 studies plus the bogus attention check study. Each study was presented and rated on a separate page. After reading the study description (and the Bayes factor plus verbal interpretation in the Description Plus Evidence condition), participants could select a tick box to indicate that they did not understand the description. Subsequently, they indicated whether they believed that this study would replicate or not (yes/no), and expressed their confidence in their decision on a slider ranging from 0 to 100 . The order in which the studies were presented was randomized across participants. ${ }^{3}$ Finally, at the end of the survey, participants were asked whether they were already familiar with the ML2 project and/or the SSRP.

\section{Data Exclusions}

Following our preregistered plan, we excluded participants (a) if they had a $\mathrm{PhD}$ in psychology (i.e., they qualified as experts rather than laypeople); (b) if they indicated that they did not understand more than 50\% of the descriptions; (c) if they did not read the descriptions carefully (i.e., they failed the included attention check); or (d) if they were already familiar with either of the replication projects from which the studies were drawn. We applied a more stringent definition of experts than did previous prediction survey studies (i.e., Camerer et al., 2018; Dreber et al., 2015; Forsell et al., 2018); whereas the previous studies defined experts as researchers in psychology, ranging from graduate students to full professors, we defined experts as people with a $\mathrm{PhD}$ degree in psychology and hence classified graduate students as laypeople. ${ }^{4}$ Participants who indicated that they had a PhD in psychology were immediately redirected to the end of the survey and could not complete the actual study. As specified in our preregistration, participants passed the attention check if they answered as explicitly instructed, that is, if they selected "no" as their answer to the dichotomous replication question and rated their confidence in the interval between $70 \%$ and $80 \%$. We excluded 3 participants because they indicated that they were familiar with the replication projects and 22 participants because they failed the attention check. No participants indicated that they understood less than $50 \%$ of the study descriptions. In total, we excluded $1.6 \%$ (i.e., 99) of all predictions for which participants indicated that

\footnotetext{
${ }^{2}$ Unfortunately, this explanation fell prey to a prevalent misinterpretation of Bayes' rule (e.g., Wagenmakers, Etz, Gronau, \& Dablander, 2018); the example describes the posterior odds (i.e., $\left.\frac{p\left(\mathcal{H}_{1} \mid \text { data }\right)}{p\left(\mathcal{H}_{0} \mid \text { data }\right)}\right)$ rather than the Bayes factor (i.e., $\frac{p\left(\text { data } \mid \mathcal{H}_{1}\right)}{\left.p \text { (data } \mathcal{H}_{0}\right)}$ ). When prior odds are assumed to be equal for the alternative and the null hypothesis -as is often assumed (e.g., Jeffreys, 1961)- the posterior odds equal the Bayes factor.

${ }^{3}$ Due to a programming error, the study descriptions were not randomized for the $n=12$ participants who were recruited from social media and selected to take the survey in Dutch.

${ }^{4}$ This discrepancy had no discernible influence on our conclusions; subsequent exploratory analyses suggested that the results did not change when excluding participants who were recruited via Amazon Mechanical Turk or social media platforms and who reported having studied psychology (at any level).
} 
they did not understand the study description. Seventy-two percent of participants (i.e., 167) understood all the study descriptions.

\section{Statistical Models}

We constructed Bayesian (hierarchical) models to estimate and test the parameters of interest for our hypotheses. For all analyses, the outcome measures were chosen according to what was most relevant and informative for answering the research questions. For the primary analysis, we estimated accuracy rates $[0-1]$ as these afford the most intuitive and simple interpretation of prediction performance and are directly comparable with the measures used in previous prediction survey studies. The experimental effect of condition (Description Only vs. Description Plus Evidence) was evaluated by means of Brier scores, because in this case the unit of interest was the individual's prediction performance, which takes into account accuracy and confidence and is the most sensitive measure for comparing people's performance across conditions. In the correlation analysis, the units of interest was the study rather than the participant; hence, here we looked at the confidence ratings per study (aggregated across participants). All models and priors are described in detail in the Supplemental Material.

\section{Results}

\section{Descriptive Pattern}

Figure 1 displays participants' confidence ratings for their predictions of the replicability of each of the 27 included studies, in order of the averaged rating. Positive ratings reflect confidence in replicability, and negative ratings reflect confidence in non-replicability; -100 denotes extreme confidence that the effect would fail to replicate. Note that these data were aggregated across the Description Only and the Description Plus Evidence condition. The studies in the top 10 rows are studies for which participants showed relatively high agreement in predicting replication success. Out of these 10 studies, 9 replicated and only 1 did not (i.e., the study by Anderson, Kraus, Galinsky, \& Keltner, 2012; note that lightgrey indicates a successful replication, and dark-grey indicates a failed replication). The studies in the bottom four rows are studies for which participants showed relatively high agreement in predicting replication failure. Consistent with laypeople's predictions, none of these four studies replicated. For the remaining 13 studies in the middle rows, the group response was relatively ambiguous, as reflected by bimodal densities that are roughly equally distributed between the negative and positive end of the scale. Out of these 13 studies, 5 replicated successfully and 8 failed to replicate successfully. Overall, Figure 1 provides a compelling demonstration that laypeople are able to predict whether or not high-profile 


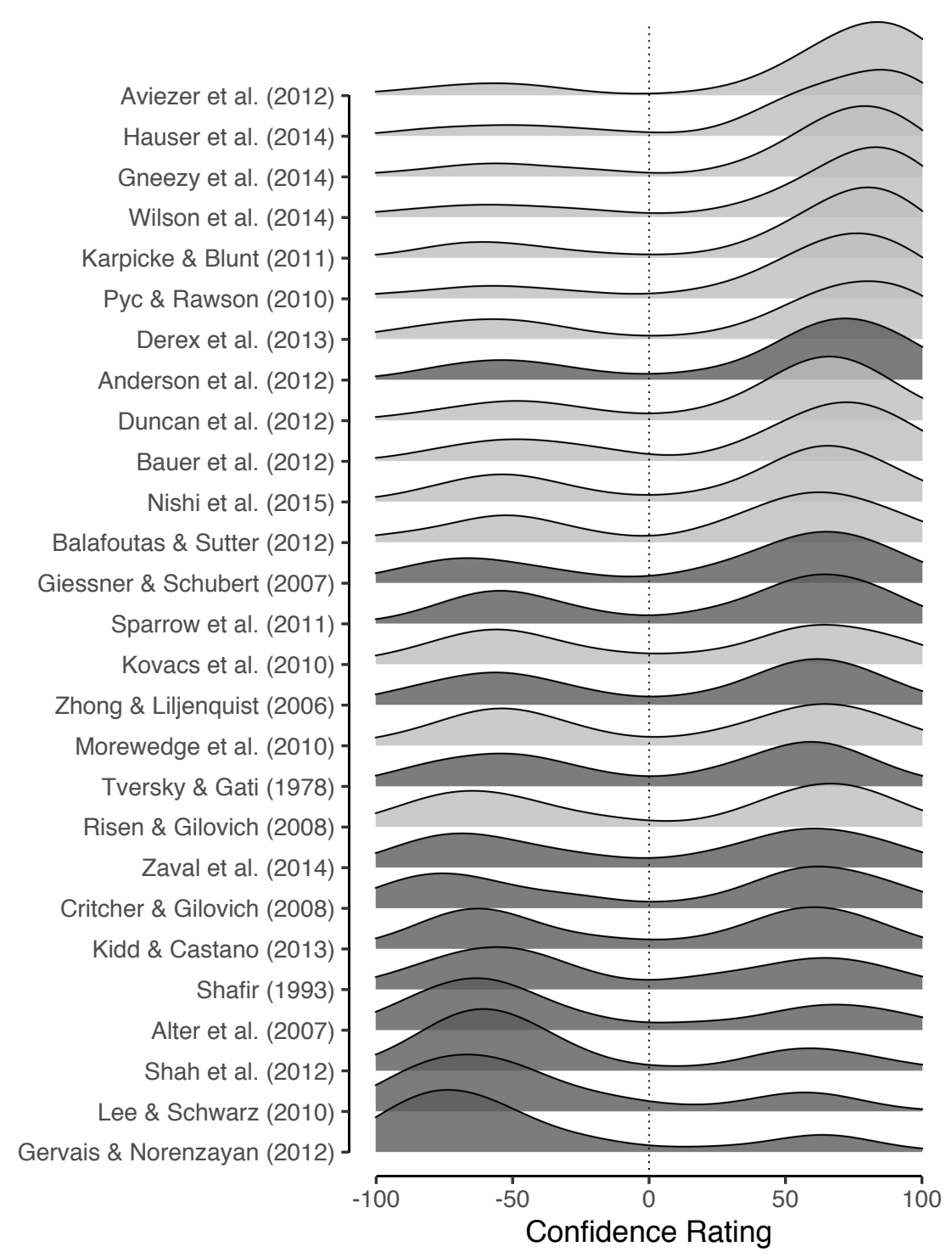

Figure 1. Laypeople's near unanimous judgments are highly predictive of replication outcomes. Light-grey shading indicates that a study successfully replicated, dark-grey shading indicates that a study did not successfully replicate. Confidence ratings are aggregated over both experimental conditions. Negative values reflect a prediction of replication failure, and positive values indicate a prediction of replication success.

social-science findings will replicate successfully. In Figure 2 participants' predictions are displayed separately for the Description Only and the Description Plus Evidence conditions.

Figure 3 provides a more detailed account of the data for three selected studies. Most participants correctly predicted that the effect found in the study in the top panel (i.e., Gneezy, Keenan, \& Gneezy, 2014) would successfully replicate. For the study in the middle panel (i.e., Tversky \& Gati, 1978), participants showed considerable disagreement; slightly 


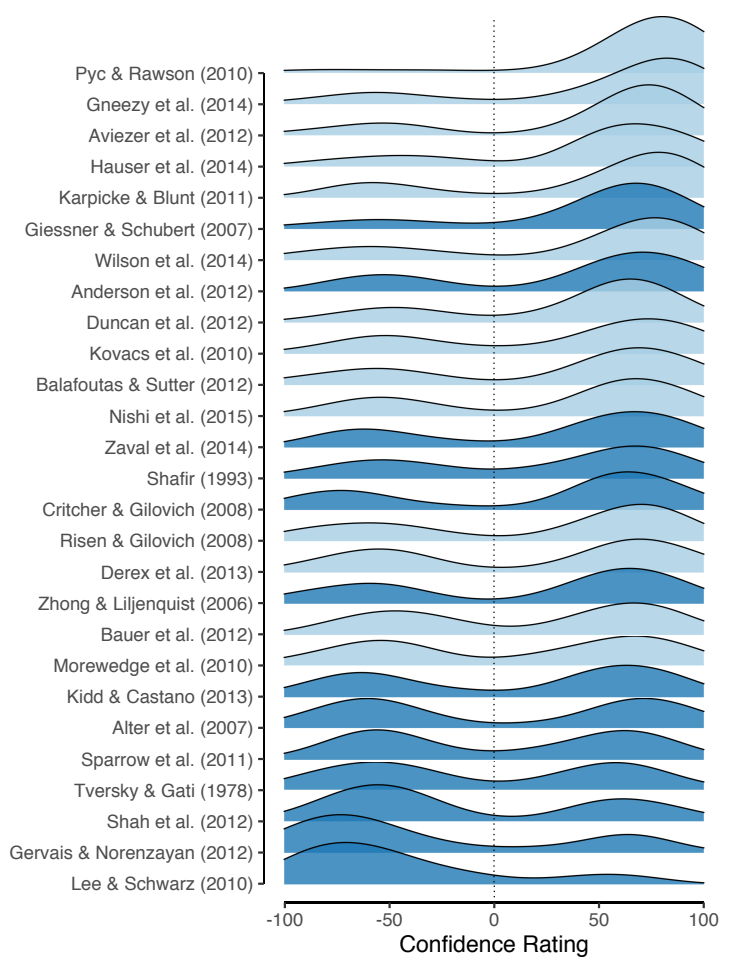

(a) Description Only condition

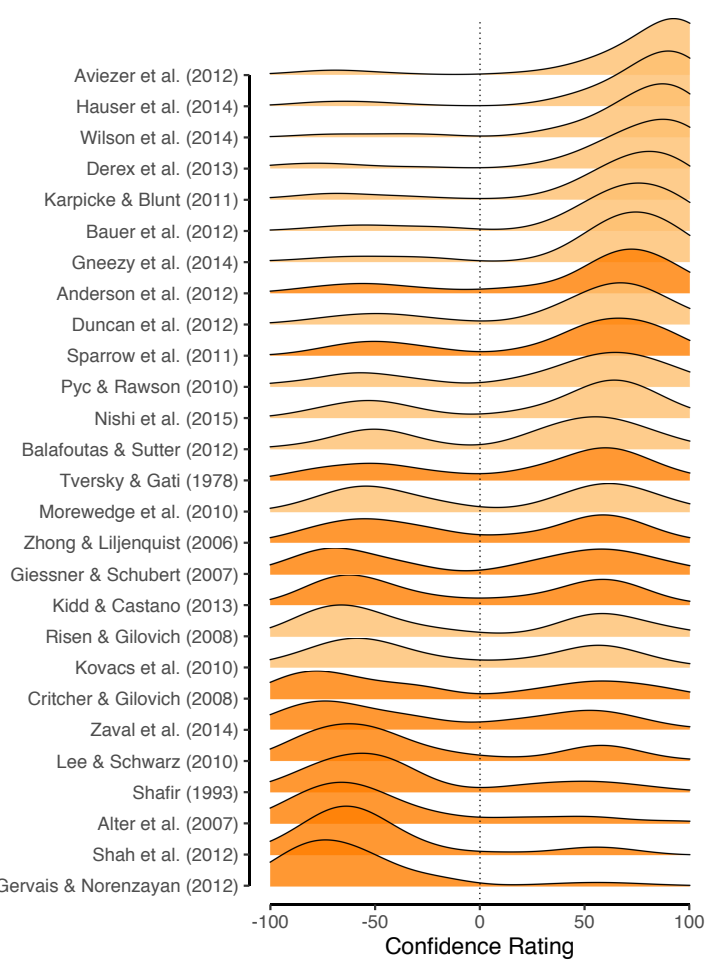

(b) Description Plus Evidence condition

Figure 2. Laypeople's predictions about replication outcomes, separately for (a) the Description Only condition and (b) the Description Plus Evidence condition. Light shading indicates that a study successfully replicated and dark shading indicates that a study did not successfully replicate. Studies are ordered according to their average confidence rating.

more than half incorrectly predicted that the study would replicate successfully. Finally, most participants correctly predicted that the effect found in the study in the bottom panel (i.e., Shah, Mullainathan, \& Shafir, 2012) would fail to replicate.

Before conducting our preregistered confirmatory analyses, we first exploratorily investigated the relation between the Bayes factors of the original studies and the effect sizes of the replication studies. To a large extent our study was based on the assumption that the Bayes factors of the original studies carry relevant information about replicability. To verify this claim we computed a Spearman correlation coefficient $\rho$ between the log-transformed Bayes factors of the original studies and the standardized effect sizes of the replication studies expressed as correlation coefficients $r$. The data provided overwhelming evidence in favor of a positive correlation $\left(\mathrm{BF}_{+0}=162\right) .{ }^{5}$ The median and $95 \%$ credible interval $(\mathrm{CI})$ for the correlation coefficient $\rho$ were $0.62[0.33,0.78]$. Thus, the Bayes factors of the original studies indeed conveyed useful information (see Figure 4).

\footnotetext{
${ }^{5}$ The subscripts on the Bayes factor to refer to the hypotheses being compared, with the first and second subscript referring to the one-sided hypothesis of interest and the null hypothesis, respectively.
} 
Gneezy et al. (2014)

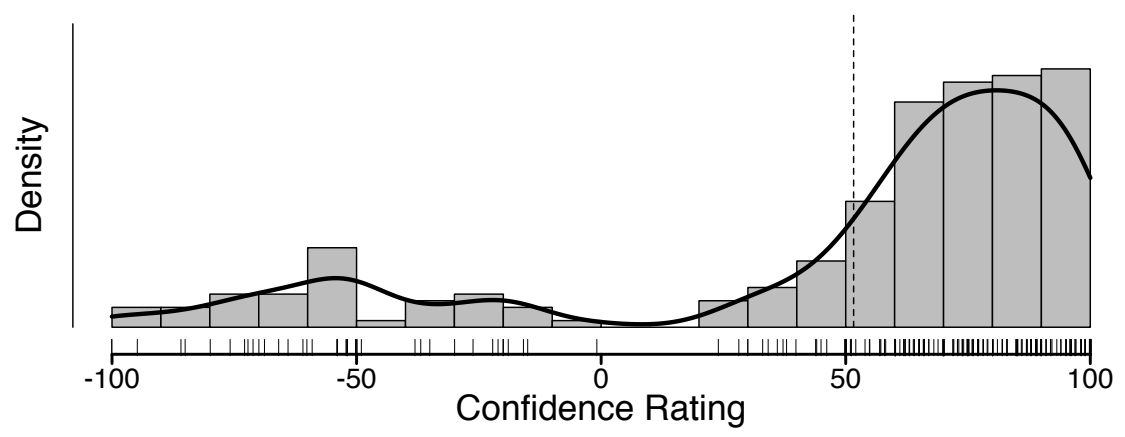

Tversky \& Gati (1978)

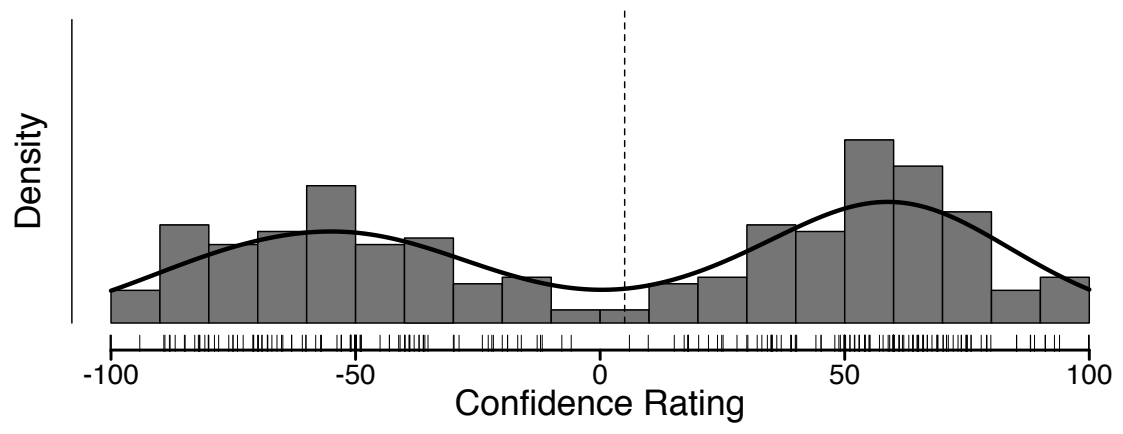

Shah et al. (2012)

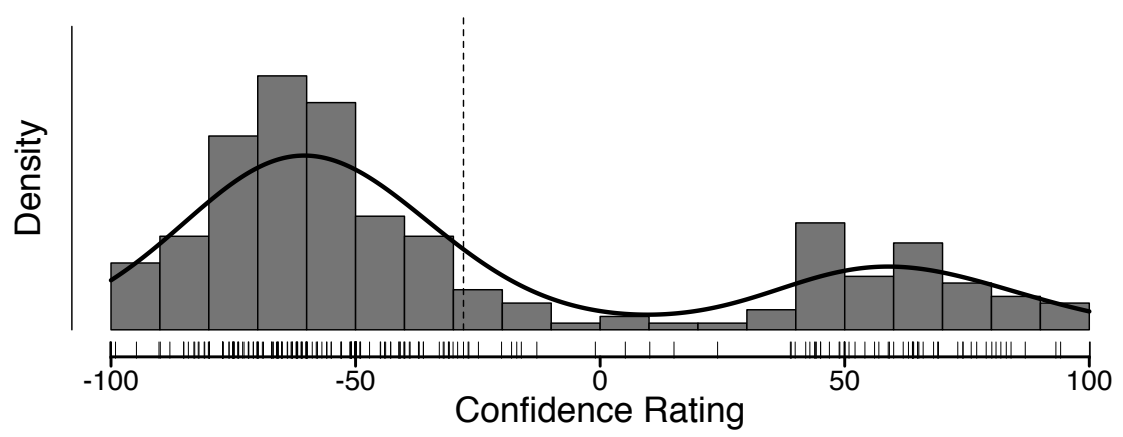

Figure 3. Histograms of confidence ratings for three selected studies, one for which participants were nearly unanimous in their belief that the study would replicate (Gneezy et al., 2014, top panel), one for which there was considerable disagreement (Tversky \& Gati, 1978, middle panel), and one for which participants were nearly unanimous in their belief that the study would not replicate (Shah et al., 2012, bottom panel). The vertical dashed lines show the average confidence ratings for the studies (i.e., group prediction).

\section{Preregistered Analyses}

\section{Quality Check}

As preregistered, we implemented a quality check on the data, which served as a prerequisite for our confirmatory analyses. We had decided to consider the data inappropriate for 


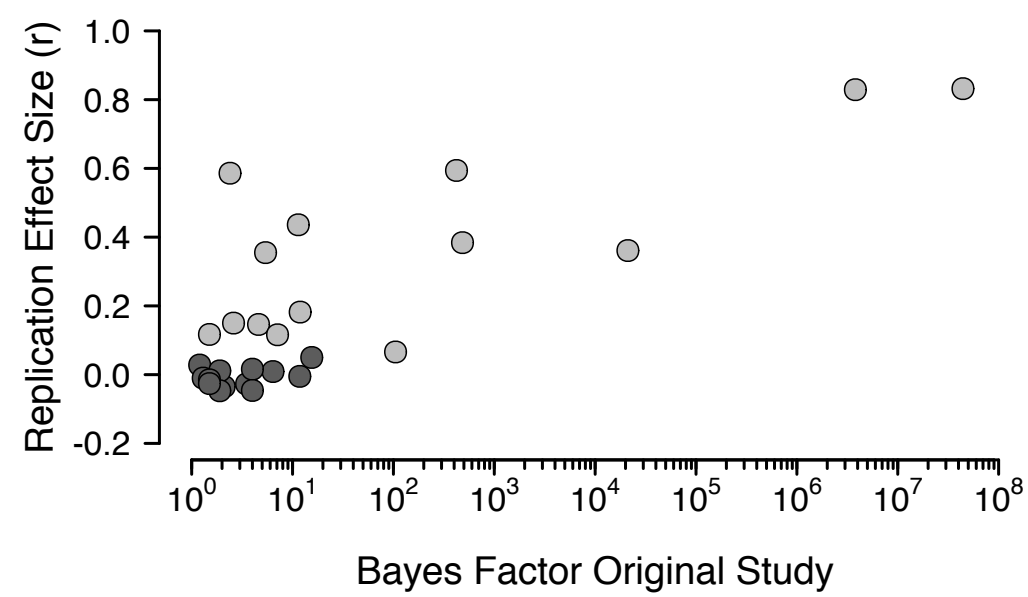

Figure 4. The evidence of the original studies (quantified by Bayes factors) is positively associated with replication effect sizes. Studies that successfully replicated are indicated by dark-grey shading and studies that did not replicate are indicated by light-grey shading.

subsequent analyses if the data provided strong evidence for the hypothesis that overall laypeople perform worse than chance level when predicting the replicability of empirical studies. An accuracy rate worse than chance level (i.e., less than 50\%) would indicate that participants did not understand or follow the instructions correctly or misinterpreted the presented information (i.e., the description of the study and the Bayes factor). We tested the restricted hypothesis $\mathcal{H}_{r 1}$ that the overall accuracy of laypeople is below $50 \%$, that is $\mathcal{H}_{r 1}: \omega<0.5$, where $\omega$ is the mode of the Beta distribution for the group-level accuracy rate. This hypothesis was tested against the encompassing hypothesis $\mathcal{H}_{e}$ which lets $\omega$ vary freely, that is $\mathcal{H}_{e}: \omega \sim \operatorname{Beta}(1,1)$. The Bayes factor in favor for the encompassing hypothesis, $\mathrm{BF}_{\text {er } 1}$, was computed using the encompassing prior approach (Klugkist, Kato, \& Hoijtink, 2005). The evidence for the encompassing hypothesis was estimated to approach infinity, that is $\mathrm{BF}_{e r 1}=\infty$, which means that the data passed the quality check. ${ }^{6}$

Difference in prediction performance between conditions. In our preregistered confirmatory analyses, we first investigated whether there was a difference between the two study conditions. Specifically, we evaluated whether or not the inclusion of the Bayes factor for the original effect increased prediction performance as measured by individual Brier scores (Brier, 1950). The Brier score takes into account both the accuracy and the indicated (un)certainty of the prediction; highly certain correct predictions are rewarded and highly certain incorrect predictions are punished, relative to uncertain predictions. Individual Brier scores were log-transformed to account for skewness in the distribution of Brier scores.

\footnotetext{
${ }^{6}$ When using the encompassing prior approach, we can obtain a Bayes factor estimated to be "infinite" if no posterior samples are in accordance with the restricted hypothesis.
} 


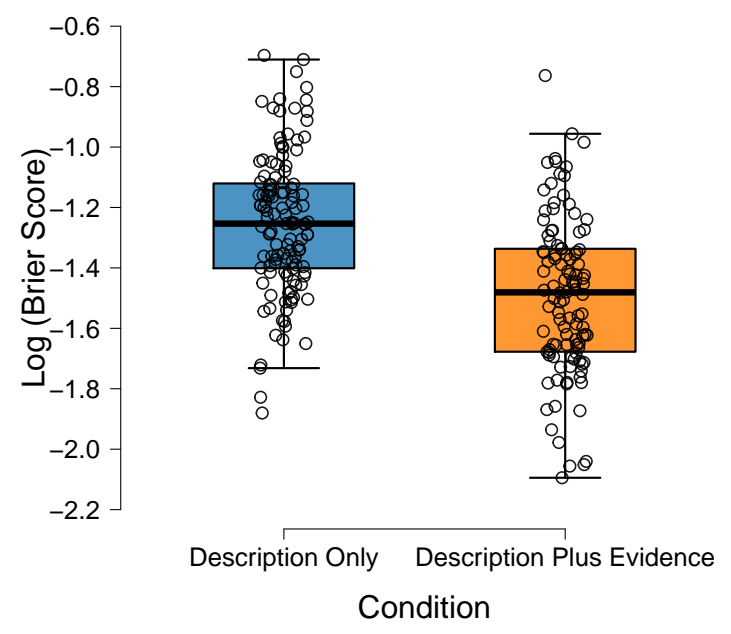

(a) Boxplot of log Brier scores per condition.

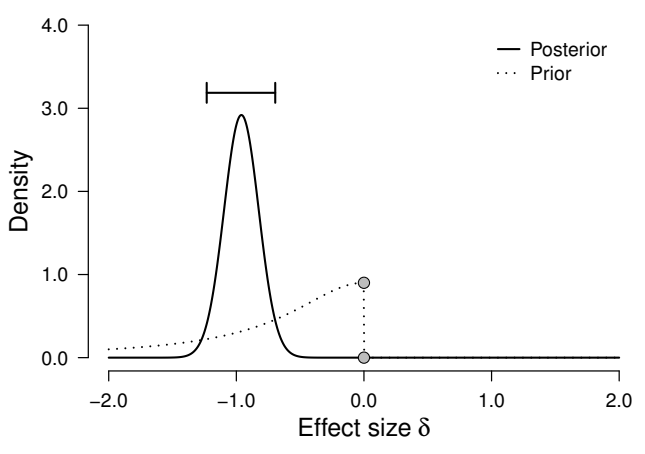

(b) Prior and posterior distribution of population effect size $\delta$.

Figure 5. The data and distribution of effect size $\delta$ of the Brier scores show that laypeople who received both the study descriptions and information about the strength of the evidence in the original study (orange boxplot) performed better than laypeople who received the study descriptions only (blue boxplot). Figure (b) shows the prior and posterior distributions of the effect size $(\delta)$.

We conducted a Bayesian independent samples $t$-test with the log Brier score as the dependent variable and the condition assignment as the grouping variable. The hypothesis of interest was that the Brier scores of participants in the Description Plus Evidence condition would be lower than the Brier scores of participants in the Description Only condition, as lower scores indicate better prediction performance. This one-sided default alternative hypothesis was specified as the effect size $\delta$ for the difference being smaller than zero, that is $\mathcal{H}-: \quad \delta<0$. The hypothesis was tested against the null hypothesis $\mathcal{H}_{0}$ that the effect size is exactly zero, that is $\mathcal{H}_{0}: \delta=0$. The results revealed overwhelming evidence that participants in the Description Plus Evidence condition outperformed those in the Description Only condition, $\mathrm{BF}_{-0}=1.0 \times 10^{10}$. The median of the effect size distribution was $-0.96,95 \% \mathrm{CI}=[-1.23,-0.68]$ (see Figure 5 for a boxplot of the data as well as the prior and posterior distributions of $\delta$ ).

Group accuracy per condition. To investigate whether laypeople can adequately predict replication outcomes, we tested whether the group-level accuracy rates ${ }^{7}$ were above chance level, that is, higher than $50 \%$. We considered only the accuracy of predictions regardless of raters' confidence. We applied a Bayesian hierarchical model to analyze the

\footnotetext{
${ }^{7}$ Note that group-level accuracy refers to the accuracy for the 'average' individual, which is estimated in a hierarchical model. A hierarchical model has the benefit that it shrinks individual estimates towards the group-level mean, thereby reducing the influence of extreme cases. Note, however, that the estimated grouplevel accuracy differs from the accuracy of the group as a collective (the latter being simply the aggregate across people per study).
} 


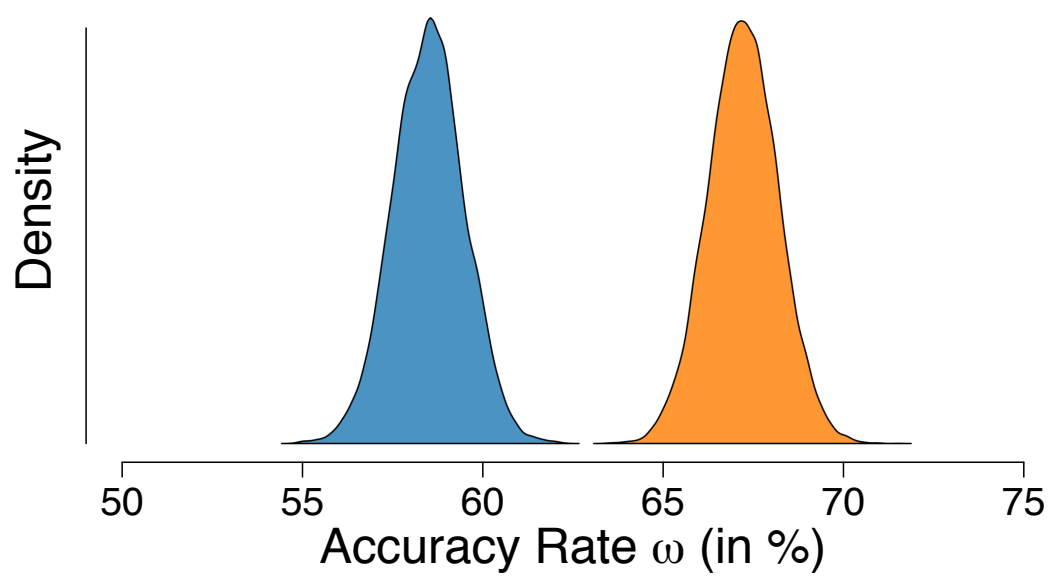

Figure 6. Accuracy rates of participants in both conditions. Posterior distributions of the group-level accuracy rate for participants in the Description Only condition are depicted in blue and those of participants in the Description Plus Evidence condition are depicted in orange.

accuracy data. For each condition separately, we then tested the restricted hypotheses that the accuracy rate $\omega$ (i.e., the mode of the group-level distribution) was above chance for laypeople in the the Description Only condition (denoted as $\mathcal{H}_{r 2}$ ), and for laypeople in the Description Plus Evidence condition (denoted as $\mathcal{H}_{r 3}$ ), that is, $\mathcal{H}_{r 2}, \mathcal{H}_{r 3}: \omega>0.5$. The hypotheses $\mathcal{H}_{r 2}$ and $\mathcal{H}_{r 3}$ were tested against the null hypothesis $\mathcal{H}_{0}$ stating that $\omega$ is exactly equal to 0.5 , which would indicate chance-level performance: $\mathcal{H}_{0}: \omega=0.5$.

The data provide extreme support for the restricted hypothesis that participants in the Description Only condition performed better than chance, $\mathrm{BF}_{r 20}=4.4 \times 10^{7}$. The median value for $\omega$ was $0.59,95 \% \mathrm{CI}=[0.57,0.60]$, which implies a $59 \%$ accuracy rate for participants in the Description Only condition at the group level. The data also provide extreme support for the restricted hypothesis that participants in the Description Plus Evidence condition performed above chance level, $\mathrm{BF}_{r 30}=5.6 \times 10^{22}$. The median value of $\omega$ was $0.67,95 \% \mathrm{CI}=[0.65,0.69]$, which implies a $67 \%$ accuracy rate for participants in the Description Plus Evidence condition at the group level. The non-overlapping credible intervals of the two conditions corroborate the results from the independent samples $t$-test on the Brier scores; accuracy was higher in the Description Plus Evidence condition than in the Description Only condition. The distributions of the accuracy rates in both conditions are displayed in Figure 6.

Correlation between laypeople's confidence and replication effect size. In addition to analyzing participants' binary predictions of replicability, we assessed whether 


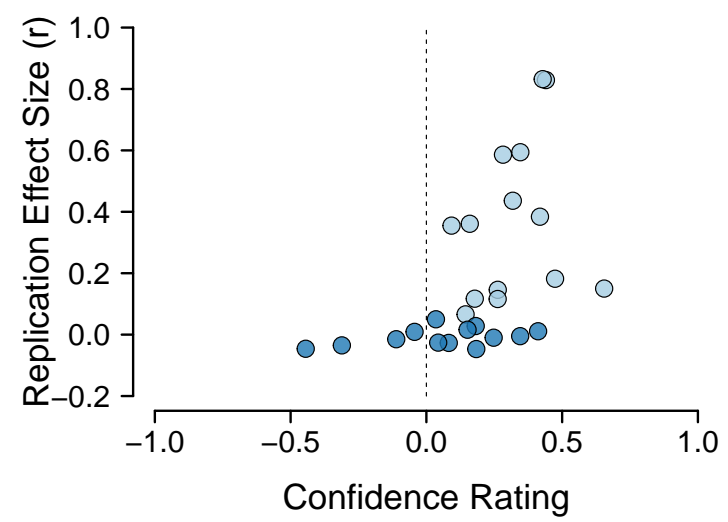

(a) Description Only condition

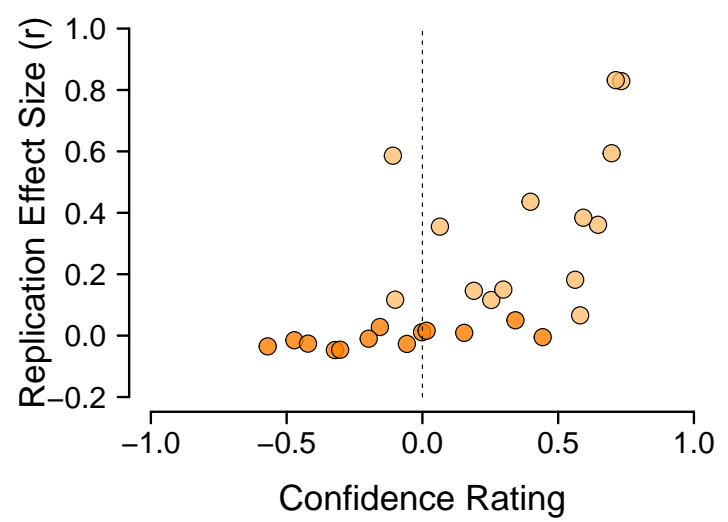

(b) Description Plus Evidence condition

Figure 7. Relationship between the average confidence rating per study and the replication effect size for (a) the Description Only condition (in blue) and (b) the Description Plus Evidence condition (in orange). The dashed lines represent the cutoff between perceived confidence in successful replication (i.e., positive values), and the perceived confidence in failed replication (i.e., negative values). Dark shading indicates that the study's effect did not replicate and the light shading indicates that the study's effect did replicate.

the confidence with which they made their decisions was indicative of the size of the effect observed in the replication studies (cf. Camerer et al., 2018). In other words, we tested whether participants were more certain about their decisions if the replication effect size iwas large, and became less certain (i.e., more certain about nonreplicability) as the underlying replication effect size approached zero. The replication effect sizes were retrieved from Camerer et al. (2018) and Klein et al. (2018). The data are plotted in Figure 7, separately for each condition.

We used a Bayesian Spearman correlation (van Doorn, Ly, Marsman, \& Wagenmakers, 2018 ) to test the null hypothesis (i.e., $\mathcal{H}_{0}: \rho=0$ ) against the one-sided restricted hypotheses that the correlation coefficient $\rho$ was positive in both the Description Only condition (i.e., $\mathcal{H}_{r 4}: \rho>0$ ) and the Description Plus Evidence condition (i.e., $\mathcal{H}_{r 5}: \rho>0$ ). The data provide extreme evidence for both restricted hypotheses, $\mathrm{BF}_{r 40}=523$ and $\mathrm{BF}_{r 50}=14,295$. For the Description Only condition the median Spearman correlation coefficient $\rho$ was 0.61, 96\% CI $=[0.34,0.77]$. For the Description Plus Evidence condition the median coefficient was $0.77,95 \% \mathrm{CI}=[0.57,0.87]$. Note that for studies that did not replicate, the effect sizes -by definition- clustered around zero. Although the Spearman correlation coefficient is a rank-based measure, the correlation should still be interpreted with caution.

\section{Exploratory Analyses}

Disentangling discriminability and response bias. According to signal detection theory (SDT; Green \& Swets, 1966; Tanner Jr \& Swets, 1954), binary decisions are 


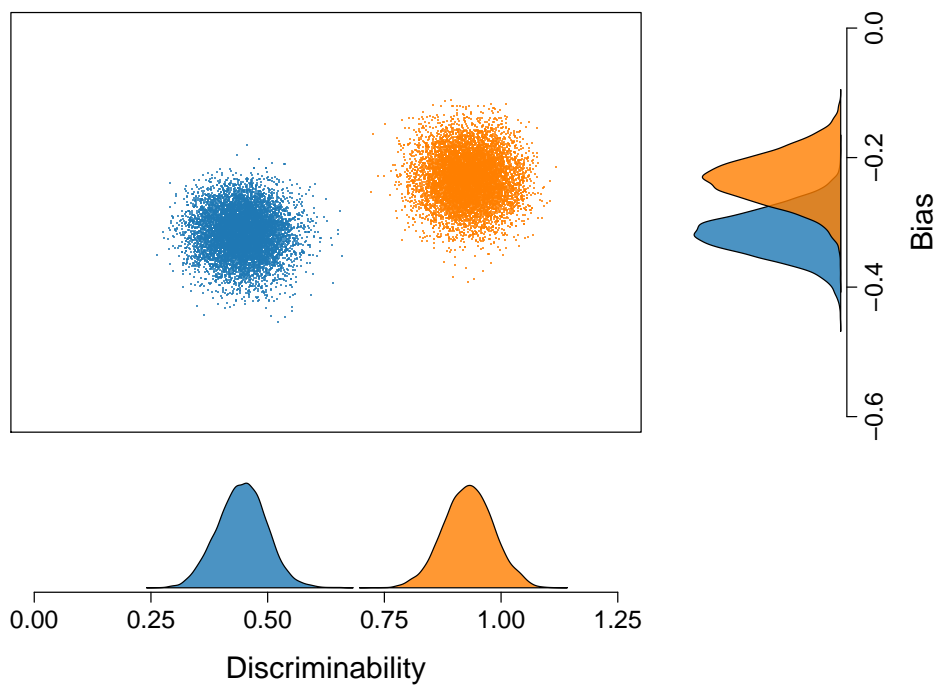

Figure 8. Laypeople in both conditions are biased towards predicting that a given study will replicate (as indicated by the posterior distributions of the bias parameter in the right panel). In addition, the posterior distributions of the discriminability parameter in the bottom panel show that laypeople in the Description Plus Evidence condition (orange) have a higher ability to correctly discriminate replicable from unreplicable studies than laypeople in the Description Only condition (blue).

driven by two main components: the ability to distinguish between the response options (discriminability) and the a priori tendency to prefer one option over the other (response bias). In an exploratory analysis, we applied SDT to decompose participants' predictions into discriminability and bias. Here, discriminability relates to the degree to which replicable and unreplicable studies were distinguishable, which was influenced by characteristics of the stimuli (i.e., information provided about the studies) and by raters' underlying ability (i.e., individual prediction skills). Bias reflects participants' overall tendency towards either predicting that a given study would replicate or predicting that it would not replicate, regardless of the information about the study. These parameters were estimated by applying a Bayesian hierarchical equal-variance Gaussian SDT model (M. D. Lee \& Wagenmakers, 2013, p. 164).

Figure 8 shows the group-level posterior distributions of the discriminability and bias parameters, separately for the two conditions. Larger values for discriminability (bottom panel) indicate greater ability to distinguish replicable from unreplicable findings. Results are consistent with the Brier score analysis reported earlier, as the discriminability parameters clearly differed between conditions; people in the Description Plus Evidence condition (blue in the figure) were better at separating replicable studies from unreplicable studies than were people in the Description Only condition (orange in the figure). The enhanced 
discriminability for the Description Plus Evidence condition is also illustrated in Figure 9a, which shows that the separation between the distribution for replicable and unreplicable studies was larger for the Description Plus Evidence condition than for the Description Only condition. For the bias parameter, the difference between conditions was less pronounced; the negative values for bias (Figure 8, right panel) indicate that all the laypeople in our sample tended to overestimate replicability (i.e., they displayed a bias towards saying that the study would replicate). This bias is also depicted in Figure 9a, in which for both conditions, the adopted criterion is located to the left of the optimal criterion.

The receiver-operating characteristic (ROC) curve is often used to interpret the parameter values of SDT. This curve reflects the proportion of hits (in this case, studies that were deemed replicable and replicated successfully) and false alarms (in this case, studies that were deemed replicable but failed to replicate) across all possible levels of bias, given the estimated discriminability. The further the curve moves away from the diagonal (i.e., chance level), the better the classification performance. The derived area under the curve (AUC) metric is used to quantify the information captured by the ROC curve; it reflects the probability that a given stimulus (in this case, study) is correctly classified (as replicable or unreplicable). We created the ROC curves for participants' prediction performance in both conditions as derived from the estimated discriminability (disregarding the estimated bias). The ROC curves in Figure 9b again show that the ratio of hits to false alarms was better for people in the Description Plus Evidence condition compared with people in the Description Only condition. This difference is also quantified by the associated AUC metric; its median value was $0.62,95 \% \mathrm{CI}=[0.60,0.65]$ for the Description Only condition and $0.74,95 \% \mathrm{CI}$ $=[0.72,0.77]$ for the Description Plus Evidence condition.

Taken together, the results of the SDT model indicate that access to the statistical evidence predominantly affected discriminability rather than bias. This suggests that the evidence (i.e., the Bayes factors) provided information that enhanced participants' ability to correctly distinguish between replicable and unreplicable studies, rather than making them simply more skeptical across the board. Note that we did not conduct any statistical tests, but solely estimated the discriminability and bias parameters for each condition, as well as the associated AUC metrics.

Estimating prediction accuracy of experts. In a second exploratory analysis, we applied a Bayesian hierarchical model to generate the posterior distributions of the accuracy rates for the experts' predictions that were obtained by Camerer et al. (2018) and Forsell et al. (2018) in the SSRP and ML2 project, respectively. Experts in the SSRP showed the higher accuracy rate; they were able to correctly predict the replicability of almost three quarters of the studies, achieving an accuracy rate of $0.72,95 \% \mathrm{CI}=[0.69,0.74]$. The median accuracy rate of the experts in the ML2 project was $0.65,95 \% \mathrm{CI}=[0.62,0.68]$. Both these 

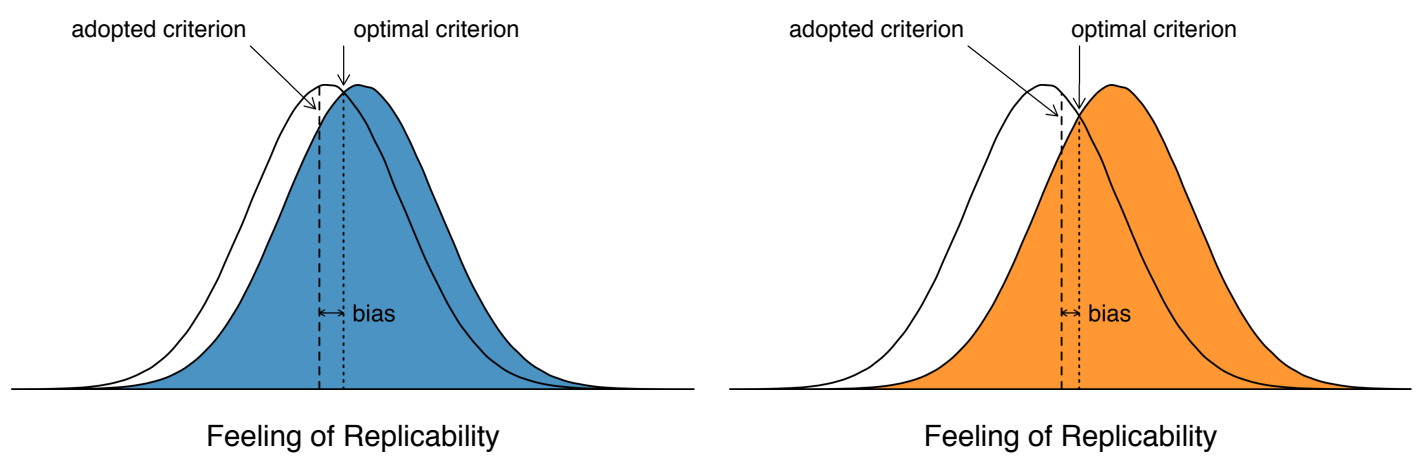

(a) Signal and noise distributions per condition
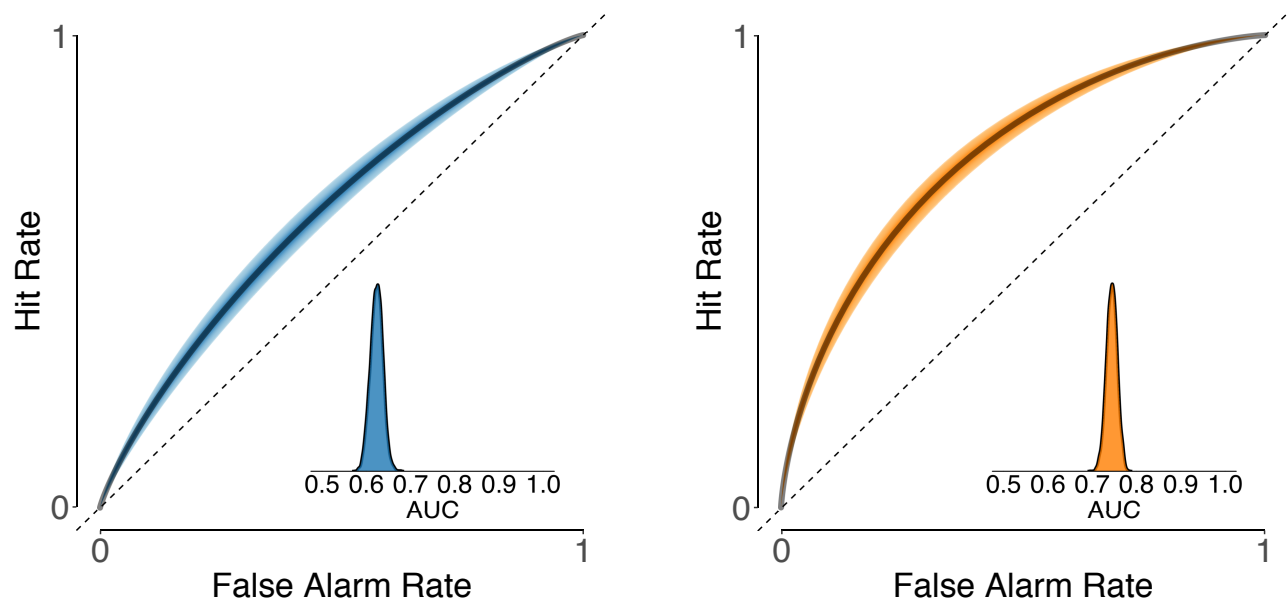

(b) Group-level ROC curves per condition

Figure 9. The top two panels demonstrate that the separation between the noise distributions (white) and signal distributions (colored) is larger for the Description Plus Evidence condition (top right panel; orange) than for the Description Only condition (top left panel; blue). The dashed lines indicate the criteria adopted by the participants and the dotted lines indicate the optimal criteria. In the bottom panels, the group-level ROC curves with the 95\% credible interval and the posterior distributions of the Area Under the Curve (AUC) metric similarly indicate that laypeople in the Description Plus Evidence condition have a better trade-off between hits and false alarms. The dashed lines indicate chance-level performance. Figure based on Figure 9 in Selker, van den Bergh, Criss, and Wagenmakers (2019).

experts' distributions and the distributions for the nonexperts in our study (expressed as percentages) are presented in Figure 10. The figure suggests that the prediction accuracy of laypeople provided with a description and a Bayes factor of the original study, can be at least as good as, if not better than, the prediction accuracy of the experts who anticipated the outcomes of the ML2 project (and who were also provided with statistics from the original studies). 


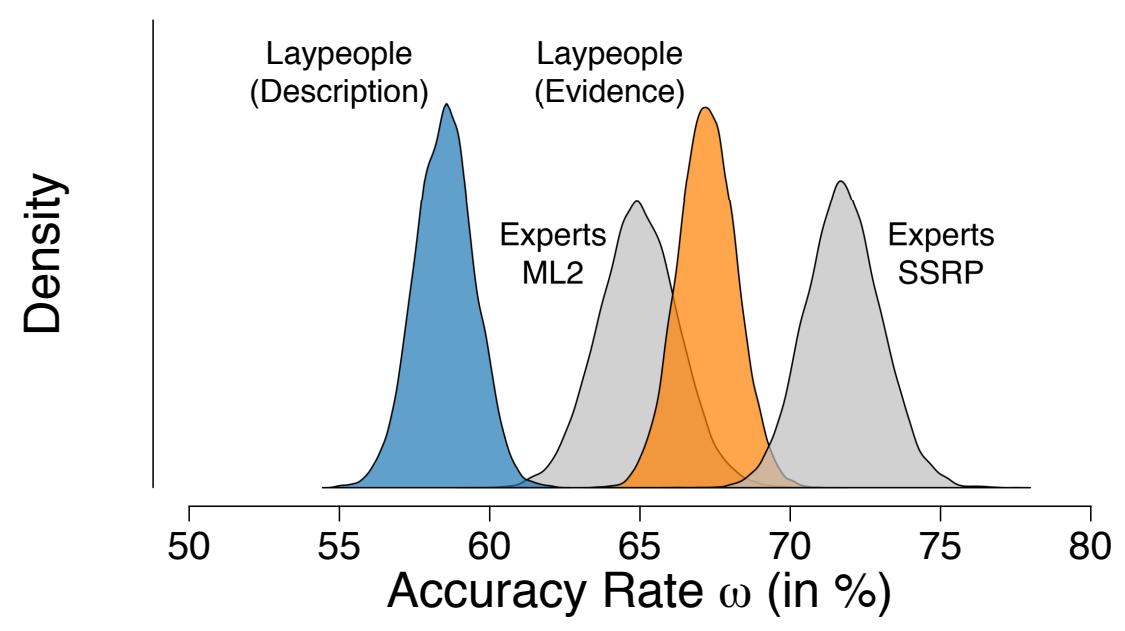

Figure 10. Accuracy rates of laypeople and experts. Posterior distributions of the grouplevel accuracy rates for laypeople in the Description Only condition are displayed in blue and for laypeople in the Description Plus Evidence condition in orange. Posterior distributions of the group-level accuracy rates for experts in the Many Labs 2 (ML2) project and in the Social Sciences Replication Project (SSRP) are displayed in grey.

It is important to note, however, that the performance of these experts and of the laypeople in our study may not be completely comparable, as the studies included in our stimuli only partly overlapped with the SSRP and ML2 sets (participants in our study rated 17 studies from the SSRP and 10 from ML2). Of the 17 studies we drew from the SSRP, 12 replicated successfully, whereas of the 10 studies we drew from ML2, only 2 replicated successfully. Because of these unintentionally unequal proportions, which are also not representative of the respective projects, we estimated the accuracy rate for the full set of studies rated by the experts in each project, rather than only the subset that we presented to our lay participants.

\section{Discussion}

This study showed that laypeople without a professional background in the social sciences are able to predict the replicability fo social-science studies with above-chance accuracy, even when provided solely with descriptions of the studies. Because the predictions were generated by nonexperts on the basis of nothing more than simple verbal study descriptions, we take these predictions to reflect intuitions of study plausibility. Thus, our results suggest that intuitions about the plausibility of targeted effects carry information about the likelihood of successful replication outcomes. Prediction accuracy further increased with access to the statistical evidence (i.e., the Bayes factors) obtained in the original studies. 
In addition, participants' confidence in their replicability judgments was associated with replication effect sizes. This may indicate that participants were able to derive a sense of the magnitude of the targeted effects from the descriptions. Inclusion of information on the original evidence amplified the relation between confidence ratings and replication effect sizes.

The notion that the intuitive plausibility of scientific effects may be indicative of their replicability is not novel (or counterintuitive). The Open Science Collaboration (2015), for instance, already suggested that nonsurprising studies are more replicable than highly surprising ones. B. M. Wilson and Wixted (2018) built on the data from the that replication project and found that lower prior odds for the crucial effects explained the difference between replicability rates in social and cognitive psychology; social psychological studies contained more risky but potentially groundbreaking effects compared with cognitivepsychological studies. The authors suggested that the key factor influencing prior odds of an effect is "established knowledge, acquired either from scientific research or from common experience (e.g., going without sleep makes a person tired)" (B. M. Wilson \& Wixted, 2018, p. 191). By asking laypeople about their intuitions regarding the replicability of socialscience studies, we sought to shed light on exactly this underlying feature of unreplicable studies derived from the latter source of knowledge, which we called "intuitive plausibility", "surprisingness", or "unexpectedness". Although we did not assess the plausibility of the studies directly, we believe that laypeople's intuitions regarding the studies' replicability can serve as a close approximation. Our results provide empirical support for the suggestion that intuitive (i.e., nonsurprising) effects are more replicable than highly surprising ones, in the sense that replicable studies were in fact deemed more replicable than nonreplicable studies by a naive group of laypeople.

The presentation of Bayes factors in the Description Plus Evidence condition could be interpreted as a demand characteristic; these quantitative markers and their verbal labels may have steered participants' judgments towards the correct conclusions. It may be practically and theoretically difficult to distinguish between demand characteristics and information given to participants in our paradigm. We do not deny that participants may have developed strategies to derive their predictions directly from the value of the Bayes factors. In fact, we assume that they did so. Although one may argue that this setup creates a confound, one can also view it as a demonstration of the benefits of Bayes factors: They constitute a simple metric that can effectively convey information about a study's evidential value. This is not a direct argument for Bayes factors over frequentist $p$-values and/or effect sizes per se; in fact, we expect that the inclusion of frequentist statistics may similarly enhance laypeople's prediction performance.

We acknowledge that replication outcomes cannot be equated with the 'truth'. Al- 
though the SSRP (Camerer et al., 2018) and Many Labs 2 (Klein et al., 2018) were highpowered projects and followed detailed preregistration protocols, the replication outcomes are not definitive or irrefutable. Moreover, there currently exists no consensus on which decision rule is superior for determining replication success (Cumming, 2008; Open Science Collaboration, 2015; Simonsohn, 2015; Verhagen \& Wagenmakers, 2014). We categorized studies as successfully replicated and not successfully replicated following the SSRP's and ML2's primary replication criteria, which were based on whether the replication study found a significant effect in the same direction as the original study. However, it should be noted that Klein et al. (2018) and Camerer et al. (2018) reported that applying additional indicators in evaluating replicability resulted in slightly different categorizations of replication success. The replication outcomes should thus be regarded not as reflective of the absolute truth, but rather as reflective of the current, tentative state of knowledge.

Along the same lines, laypeople's predictions should also not be equated with the truth. Although clearly above chance level, the prediction accuracy rates of $59 \%$ and $67 \%$ in the Description Only and the Description Plus Evidence conditions, respectively, are far from perfect. One reason for this moderate prediction success may be participants' tendency to overestimate the replicability of empirical findings; relative to the bleak reality of the current replication rate in psychological science, laypeople are optimists. This pattern is evident in Figure 1 and is corroborated by the signal detection analysis indicating that our participants had a bias toward saying that a given study would replicate. Notably, the optimistic perspective does not seem to be unique to laypeople; experts similarly overestimated replicability in Dreber et al. (2015), Camerer et al. (2016) and Forsell et al. (2018), though not in Camerer et al. (2018). This biased responding may allow for boosting prediction accuracy; the AUC metric in our project indicates that if laypeople adopted the optimal unbiased criterion, that is, if they were more conservative, then their accuracy might be enhanced to $62 \%$ for predictions based on verbal descriptions only and $74 \%$ for predictions based on descriptions plus evidence from the original study. This suggestion is speculative, but it could be tested in future research, for instance by manipulating expectations of baseline replicability rates.

Nevertheless, we believe that laypeople's predictions provide more information than is captured by the estimated accuracy rates. This is exemplified by the prediction pattern as displayed in Figure 1. The pattern suggests that participants as a collective were divided for a group of studies (i.e, those in the middle rows, with symmetrical bimodal distributions) and were in agreement for another group (i.e., those in the top and bottom rows of the figure). For those studies for which participants were nearly unanimous, the predictions were highly accurate. Moreover, as the figure shows, when participants as a group predicted that a particular effect would fail to replicate, it indeed failed to replicate. 
These results indicate that the scientific culture of striving for newsworthy, extreme, and sexy findings is indeed problematic, as counterintuitive findings are the least likely to replicate. Our results also relate to the aphorism that "extraordinary (i.e., intuitively implausible) claims require extraordinary evidence". Many studies included in our sample were considered implausible and thus would have required highly compelling evidence for the effects to be established. However, the pattern of Bayes factors in Figure 4 shows that many of the effects in the original studies were based on weak initial evidence; of the included studies, 37\% (10 studies) yielded a Bayes factor lower than 3, evidence that is "not worth more than a bare comment" according to Jeffreys' (1939, p.357) criteria. The combination of low intuitive plausibility and weak initial evidence is remarkable and arguably worrisome, especially in light of the low replication rates in social science. To account for the extraordinary nature of a claim, researchers should adjust the prior probability of the alternative hypothesis and the null hypothesis. In the Bayesian framework, this means that a higher Bayes factor would be necessary to conclude that the effect is present; in the frequentist framework, a lower $p$-value would be necessary to reject the null hypothesis (cf. Benjamin et al., 2018).

The notion of prediction surveys and markets as a valuable component of replication research seems to be gaining momentum. The Replication Markets platform (https://www.replicationmarkets.com), for instance, invites researchers as well as the general public to predict and bet on 3,000 studies associated with the SCORE project (https://www.darpa.mil/program/systematizing-confidence-in -open-research-and-evidence). Although these predictions yield valuable insights, we naturally do not advocate replacing replication studies with judgments of the general public - or with those of experts. Rather, laypeople's predictions may be used to provide a quick snapshot of expected replicability. This can facilitate the replication process by informing the selection of to-be-replicated studies. The uni- versus bimodality of the distribution of laypeople's replication predictions may for instance steer researchers' confidence in evaluating which predictions are more or less reliable, respectively. Additionally, the relative ordering of laypeople's confidence in the replicability of a given set of studies may provide estimations of the relative probabilities of replication success. If the goal is to purge the literature of unreliable effects, one may start by conducting replications of the studies for which replication failure is predicted by naive forecasters. Alternatively, if the goal is to clarify the reliability of studies for which replication outcomes are most uncertain, one could select studies for which the distribution of the expected replicability is bimodal. As such, prediction surveys may serve as 'decision surveys', instrumental in the selection stage of replication research (cf. Dreber et al., 2015). These informed decisions not only could benefit the researchers attempting replications, but also could optimize the distribution 
of funds and resources for replication projects. In addition, the application of prediction surveys to assess an effect's prior plausibility can easily be extended to proposed and yetto-be-empirically-investigated hypotheses systematically, as is done with the Social Science Prediction Platform (https://socialscienceprediction.org/). An interesting application would be to use these assessments in conjunction with large collaborative research efforts such as the Psychological Science Accelerator (Moshontz et al., 2018). In this way, laypeople's predictions may not only contribute to replication research, but may also inform the prior plausibility of novel studies.

\section{Constraints on Generality}

In principle, we expect our results to generalize to most people, provided that the instructions, explanation of replicability, and study descriptions are written in plain language, avoiding technical terms. It is possible that prediction accuracy may rise with increased expertise; for instance, graduate students may on average outperform people without any expertise in social sciences. However, previous prediction studies showed that weighting experts' predictions based on self-reported topical expertise did not improve their average prediction accuracy, which suggests that at least knowledge about a particular study's topic may be irrelevant (Dreber et al., 2015; Forsell et al., 2018). An obvious limitation of prediction surveys that solicit predictions from laypeople, is that it narrows the pool of studies that are suited for inclusion; complex psychophysics experiments or functional MRI studies may not be comprehensible for laypeople and may be better evaluated by experts. However, for the majority of studies in social science (e.g., psychology and economics) targeting laypeople rather than experts may be advantageous in terms of availability, accessibility, and the possibility of including previously published studies (the results of which experts may already be familiar with or simply look up). A further prerequisite is that the evaluated replication studies should be of high quality (e.g., preregistered, high-powered studies featuring manipulation checks) to ensure that the replication outcomes are reliable and can thus serve as a reliable criterion to compare participants' predictions to. We have no reason to believe that our results depend on other characteristics of the participants, materials, or context. A final side-note on the generalizability of the findings concerns the wider implications and scope of the results. Although participants in our study strongly overestimated overall replicability, they still believed that approximately $20 \%$ of the studies would not replicate. This does not necessarily imply, however, that they will distrust the results of 1 in 5 studies they encounter in the media. 


\section{Author Contributions}

S. Hoogeveen, A. Sarafoglou, and E.J. Wagenmakers jointly generated the idea for the study. S. Hoogeveen and A. Sarafoglou wrote the preregistration, constructed the survey, collected and analyzed the data, and wrote the first draft of the manuscript. All three authors critically edited the manuscript. All authors approved the final submitted version of the manuscript.

\section{Conflicts of Interest}

The authors declared that there were no conflicts of interest with respect to the authorship or the publication of this article.

\section{Acknowledgements}

We thank Job Bank, Chiel van 't Hoff, and Philip Kleinbrahm for their support in creating the study materials and collecting the data.

\section{Funding}

This research was supported by a talent grant from the Netherlands Organisation for Scientific Research (NWO) to AS (406-17-568), as well as a Vici grant from the NWO to EJW (016.Vici.170.083).

\section{Supplemental Material}

Additional supporting information can be found at https://osf.io/7cgfw/.

\section{References}

Alter, A. L., Oppenheimer, D. M., Epley, N., \& Eyre, R. N. (2007). Overcoming intuition: Metacognitive difficulty activates analytic reasoning. Journal of Experimental Psychology: General, $136,569-576$.

Anderson, C., Kraus, M. W., Galinsky, A. D., \& Keltner, D. (2012). The local-ladder effect: Social status and subjective well-being. Psychological Science, 23, 764-771. doi: 10.1177/ 0956797611434537

Aviezer, H., Trope, Y., \& Todorov, A. (2012). Body cues, not facial expressions, discriminate between intense positive and negative emotions. Science, 338, 1225-1229.

Baker, M. (2016). Is there a reproducibility crisis? Nature, 533, 452-454.

Balafoutas, L., \& Sutter, M. (2012). Affirmative action policies promote women and do not harm efficiency in the laboratory. Science, 335, 579-582.

Bauer, M. A., Wilkie, J. E., Kim, J. K., \& Bodenhausen, G. V. (2012). Cuing consumerism: Situational materialism undermines personal and social well-being. Psychological Science, 23, $517-523$. 
Benjamin, D. J., Berger, J. O., Johannesson, M., Nosek, B. A., Wagenmakers, E.-J., Berk, R., ... Johnson, V. E. (2018). Redefine statistical significance. Nature Human Behaviour, 2, 6-10.

Brier, G. W. (1950). Verification of forecasts expressed in terms of probability. Monthly Weather Review, 78, 1-3.

Button, K. S., Ioannidis, J. P. A., Mokrysz, C., Nosek, B. A., Flint, J., Robinson, E. S. J., \& Munafò, M. R. (2013). Power failure: Why small sample size undermines the reliability of neuroscience. Nature Reviews Neuroscience, 14, 365-376. doi: 10.1038/nrn3475

Camerer, C. F., Dreber, A., Ho, T.-H., Huber, J., Johannesson, M., Kirchler, M., ... Razen, M. (2016). Evaluating replicability of laboratory experiments in economics. Science, 351, 14331436. doi: $10.1126 /$ science.aaf0918

Camerer, C. F., Dreber, A., Holzmeister, F., Ho, T.-H., Huber, J., Johannesson, M., ... Wu, H. (2018). Evaluating replicability of social science experiments in Nature and Science. Nature Human Behaviour, 2, 637-644.

Chambers, C. D. (2013). Registered reports: A new publishing initiative at Cortex. Cortex, 49, 609-610.

Critcher, C. R., \& Gilovich, T. (2008). Incidental environmental anchors. Journal of Behavioral Decision Making, 21, 241-251.

Cumming, G. (2008). Replication and $p$ intervals: $p$-values predict the future only vaguely, but confidence intervals do much better. Perspectives on Psychological Science, 3, 286-300. doi: 10.1111/j.1745-6924.2008.00079.x

Derex, M., Beugin, M.-P., Godelle, B., \& Raymond, M. (2013). Experimental evidence for the influence of group size on cultural complexity. Nature, 503, 389-391.

Dovidio, J. F. (2016). Commentary: A big problem requires a foundational change. Journal of Experimental Social Psychology, 66, 159-165. doi: 10.1016/j.jesp.2016.01.008

Dreber, A., Pfeiffer, T., Almenberg, J., Isaksson, S., Wilson, B., Chen, Y., ... Johannesson, M. (2015). Using Prediction Markets to Estimate the Reproducibility of Scientific Research. Proceedings of the National Academy of Sciences of the United States of America, 112, 1534315347. doi: 10.1073/pnas.1516179112

Duncan, K., Sadanand, A., \& Davachi, L. (2012). Memory's penumbra: Episodic memory decisions induce lingering mnemonic biases. Science, 337, 485-487.

Etz, A., Bartlema, A., Vanpaemel, W., Wagenmakers, E.-J., \& Morey, R. D. (2019). An exploratory survey of student and researcher intuitions about statistical evidence. Poster presented at the 31st annual convention of the Association of Psychological Science (APS), Washington, USA.

Forsell, E., Viganola, D., Pfeiffer, T., Almenberg, J., Wilson, B., Chen, Y., ... Dreber, A. (2018). Predicting replication outcomes in the Many Labs 2 study. Journal of Economic Psychology. doi: $10.1016 /$ j.joep.2018.10.009

Francis, G. (2013). Replication, Statistical Consistency, and Publication Bias. Journal of Mathematical Psychology, 57, 153-169. doi: 10.1016/j.jmp.2013.02.003

Gervais, W. M., \& Norenzayan, A. (2012). Analytic thinking promotes religious disbelief. Science, 336, 493-496.

Giessner, S. R., \& Schubert, T. W. (2007). High in the hierarchy: How vertical location and judgments of leaders' power are interrelated. Organizational Behavior and Human Decision 
Processes, 104, 30-44.

Giner-Sorolla, R. (2012). Science or art? How aesthetic standards grease the way through the publication bottleneck but undermine science. Perspectives on Psychological Science, 7, 562571. doi: $10.1177 / 1745691612457576$

Gneezy, U., Keenan, E. A., \& Gneezy, A. (2014). Avoiding Overhead Aversion in Charity. Science, 346, 632-635. doi: 10.1126/science.1253932

Green, D. M., \& Swets, J. A. (1966). Signal Detection Theory and Psychophysics. New York: Wiley.

Hardwicke, T. E., Tessler, M. H., Peloquin, B., \& Frank, M. C. (2018). A Bayesian decisionmaking framework for replication. Behavioral and Brain Sciences, 41, e132. doi: 10.1017/ S0140525X18000675

Hauser, O. P., Rand, D. G., Peysakhovich, A., \& Nowak, M. A. (2014). Cooperating with the future. Nature, 511, 220-223.

Ioannidis, J. P. A. (2005). Why most published research findings are false. PLoS Medicine, 2, 696-701. doi: 10.1080/09332480.2005.10722754

JASP Team. (2019). JASP (Version 0.11.1)[Computer software]. Retrieved from https://jasp -stats.org/

Jeffreys, H. (1939). Theory of Probability (1st ed.). Oxford, UK: Oxford University Press.

Jeffreys, H. (1961). Theory of Probability (3rd ed.). Oxford, UK: Oxford University Press.

John, L. K., Loewenstein, G., \& Prelec, D. (2012). Measuring the prevalence of questionable research practices with incentives for truth-telling. Psychological Science, 23, 524-532. doi: 10.1037/e632032012-001

Karpicke, J. D., \& Blunt, J. R. (2011). Retrieval practice produces more learning than elaborative studying with concept mapping. Science, 331, 772-775.

Kass, R. E., \& Raftery, A. E. (1995). Bayes factors. Journal of the American Statistical Association, 90, 773-795. doi: 10.2307/2291091

Kidd, D. C., \& Castano, E. (2013). Reading Literary Fiction Improves Theory of Mind. Science, 342, 377-380. doi: 10.1126/science.1239918

Klein, R., Ratliff, K., Vianello, M., Adams, R. B., Jr., Bahník, v., Bernstein, M., .. Nosek, B. (2014). Investigating variation in replicability: A "Many Labs" replication project. Social Psychology, 45, 142-152. doi: 10.1027/1864-9335/a000178

Klein, R., Ratliff, K., Vianello, M., Adams Jr, R., Bahník, Š., Bernstein, M. J., .. Nosek, B. A. (2014). Theory building through replication: Response to commentaries on the "Many Labs" replication project. Social Psychology, 45, 299-311.

Klein, R., Vianello, M., Hasselman, F., Adams, B., Adams, R., Alper, S., .. Nosek, B. (2018). Many Labs 2: Investigating variation in replicability across sample and setting. Advances in Methods and Practices in Psychological Science, 1, 443-490.

Klugkist, I., Kato, B., \& Hoijtink, H. (2005). Bayesian model selection using encompassing priors. Statistica Neerlandica, 59, 57-69. doi: 10.1111/j.1467-9574.2005.00279.x

Kovacs, A. M., Téglás, E., \& Endress, A. D. (2010). The social sense: Susceptibility to others' beliefs in human infants and adults. Science, 330, 1830-1834.

Lee, M. D., \& Wagenmakers, E.-J. (2013). Bayesian Cognitive Modeling: A Practical Course. Cambridge University Press. 
Lee, S. W. S., \& Schwarz, N. (2010). Washing away postdecisional dissonance. Science, 328, 709.

MacKay, D. J. (1992). Information-based objective functions for active data selection. Neural Computation, 4, 590-604. doi: 10.1162/neco.1992.4.4.590

Milkman, K. L., \& Berger, J. (2014). The science of sharing and the sharing of science. Proceedings of the National Academy of Sciences, 111, 13642-13649. doi: 10.1073/pnas.1317511111

Morewedge, C. K., Huh, Y. E., \& Vosgerau, J. (2010). Thought for food: Imagined consumption reduces actual consumption. Science, 330, 1530-1533.

Moshontz, H., Campbell, L., Ebersole, C. R., IJzerman, H., Urry, H. L., Forscher, P. S., . . Chartier, C. R. (2018). The Psychological Science Accelerator: Advancing psychology through a distributed collaborative network. Advances in Methods and Practices in Psychological Science, 1, 501-515. doi: $10.1177 / 2515245918797607$

Nishi, A., Shirado, H., Rand, D. G., \& Christakis, N. A. (2015). Inequality and visibility of wealth in experimental social networks. Nature, 526, 426-429.

Nosek, B. A., Spies, J. R., \& Motyl, M. (2012). Scientific Utopia: II. Restructuring incentives and practices to promote truth over publishability. Perspectives on Psychological Science, 7, 615-631. doi: 10.1177/1745691612459058

Open Science Collaboration, T. (2015). Estimating the reproducibility of psychological science. Science, 349, aac4716. doi: 10.1126/science.aac4716

Pyc, M. A., \& Rawson, K. A. (2010). Why testing improves memory: Mediator effectiveness hypothesis. Science, 330, 335.

Risen, J. L., \& Gilovich, T. (2008). Why people are reluctant to tempt fate. Journal of Personality and Social Psychology, 95, 293-307.

Selker, R., van den Bergh, D., Criss, A. H., \& Wagenmakers, E.-J. (2019). Parsimonious estimation of signal detection models from confidence ratings. Behavior Research Methods. doi: 10.3758/ s13428-019-01231-3

Shafir, E. (1993). Choosing versus rejecting: Why some options are both better and worse than others. Memory \& Cognition, 21, 546-556.

Shah, A. K., Mullainathan, S., \& Shafir, E. (2012). Some consequences of having too little. Science, 338, 682-685. doi: 10.1126/science.1222426

Simmons, J. P., Nelson, L. D., \& Simonsohn, U. (2011). False-positive psychology: Undisclosed flexibility in data collection and analysis allows presenting anything as significant. Psychological Science, 22, 1359-1366. doi: 10.1037/e519702015-014

Simonsohn, U. (2015). Small telescopes: Detectability and the evaluation of replication results. Psychological Science, 26, 559-569. doi: 10.1177/0956797614567341

Sparrow, B., Liu, J., \& Wegner, D. M. (2011). Google effects on memory: Cognitive consequences of having information at our fingertips. Science, 333, 776-778.

Tanner Jr, W. P., \& Swets, J. A. (1954). A decision-making theory of visual detection. Psychological Review, 61, 401-409. doi: 10.1037/h0058700

Tversky, A., \& Gati, I. (1978). Studies of similarity. Cognition and Categorization, 1, 79-98.

van Doorn, J., Ly, A., Marsman, M., \& Wagenmakers, E.-J. (2018). Bayesian latent-normal inference for the rank sum test, the signed rank test, and Spearman's $\rho$. Preprint via https:// arxiv.org/abs/1712.06941. 
Verhagen, A. J., \& Wagenmakers, E.-J. (2014). Bayesian ests to quantify the result of a replication attempt. Journal of Experimental Psychology: General, 143, 1457-1475. doi: $10.1037 / \mathrm{a} 0036731$

Wagenmakers, E.-J., Etz, A., Gronau, Q., \& Dablander, F. (2018). The single most prevalent misinterpretation of Bayes' rule [Blog Post]. https://www.bayesianspectacles.org/the-singlemost-prevalent-misinterpretation-of-bayes-rule/.

Wagenmakers, E.-J., Wetzels, R., Borsboom, D., \& van der Maas, H. L. J. (2011). Why psychologists must change the way they analyze their data: The case of psi: Comment on Bem (2011). Journal of Personality and Social Psychology, 100, 426-432. doi: 10.1037/a0022790

Wilson, B. M., \& Wixted, J. T. (2018). The prior odds of testing a true effect in cognitive and social psychology. Advances in Methods and Practices in Psychological Science, 1, 186-197. doi: $10.1177 / 2515245918767122$

Wilson, T. D., Reinhard, D. A., Westgate, E. C., Gilbert, D. T., Ellerbeck, N., Hahn, C., .. Shaked, A. (2014). Just think: The challenges of the disengaged mind. Science, 345, 75-77.

Zaval, L., Keenan, E. A., Johnson, E. J., \& Weber, E. U. (2014). How warm days increase belief in global warming. Nature Climate Change, 4, 143-147.

Zhong, C.-B., \& Liljenquist, K. (2006). Washing away your sins: Threatened morality and physical cleansing. Science, 313, 1451-1452. 